\title{
Shallow foundation analysis using the discrete element method
}

\begin{abstract}
This research presents a numerical evaluation of shallow foundation using the discrete element method and parameters calibrated by means of a reduced laboratory model of an idealized soil. For this evaluation, the Yade software was used to analyze the influence of grain scale parameters (mesoscale) on the macroscopic behavior of the soil, perceived as an assembly of isolated and interacting particles. It was observed that, despite the need for calibration, the discrete element method showed an appropriate qualitative prediction of failure surfaces and a satisfactory quantitative prediction of ultimate load capacity.
\end{abstract}

Keywords: discrete element method, shallow foundation, reduced model
Volume 3 Issue 4 - 2019

\author{
Jéssica Soares da Rocha, Márcio Muniz de \\ Farias, Bernardo Cascão Pires e Albuquerque, \\ Joaquim Araújo Costa Neto \\ Department of Civil Engineering, University of Brasilia, Brazil
}

Correspondence: Joaquim Araújo Costa Neto, Department of Civil Engineering, University of Brasilia, CLN 4 I I bloco C, Brasilia, Distrito Federal, Brasil, Email engcjoaquim@gmail.com

Received: July 20, 2019 | Published: August 08, 2019
Abbreviations: DEM, discrete element method; FEM, finite element method; NIT, Nagoya Institute of Technology

\section{Introduction}

The use of physical models in full or reduced scale in the past was one of the main tools to predict the behavior of civil and mechanical engineering constructions and structures. ${ }^{1}$ With the increasing costs of model construction, in part because of the high prices of instrumentation that became increasingly sophisticated and accurate, and also the difficulty to extrapolate quantitative results of reduced analyses, physical models were gradually being less used. Alternatively, computational development provided a greater use of numerical methods for the simulation of these models, with the finite element method (FEM) having the most practical impact in the last decades. However, it is necessary to evaluate if these models are able to predict the behavior of the problem under study, because when addressing the behavior of the soil as a continuous material, certain constraints are imposed in the analyzes performed. In addition, this approach implies the need to formulate phenomenological constitutive models that study the behavior of materials in a macroscopic or phenomenological way. ${ }^{2}$ A macroscopic approach, on the other hand, does not consider the properties of the soil in the grain scale, which may result in an incomplete understanding of its behavior or in an unreasonable approximation for granular soils or highly fractured media. ${ }^{3}$ In an attempt to consider the basic phenomena, a new numerical approach that considers the mesoscale aspects in the mechanical model of the soil was developed. This numerical method is known as the discrete element method (DEM) and it is based on the discretization of particles for modeling and problem solving. ${ }^{4}$ The application of DEM to simulate small scale problems is an intermediate option to evaluate the mechanisms in a geotechnical analysis. Thus, reduced models properly instrumented and allied to a discrete numerical simulation can be an option with great potential for qualitative and quantitative prediction of geotechnical constructions. The motivation of this research consists in the possibility of improving the understanding of the behavior of shallow foundation, using the DEM associated with reduced model simulations to evaluate the effectiveness of this method to predict shallow foundation (block) behavior.

\section{Discrete element method}

The discrete element method is a numerical technique proposed by Cundall $\&$ Strack $^{5}$ by which the analyzed medium is constituted by a set of particles with fundamental mechanical properties and defined geometry. In addition, it uses primary physical variables such as contact forces, momentum, displacement and rotation. This method is suitable for the study of particulate medium, since it explicitly considers its discrete nature. Moreover, it is possible to evaluate the physical and mechanical behavior of granular materials by understanding the mechanical properties of particles and their interactions. $^{2}$

The DEM consists of a transient analysis that considers the dynamic interactions between a system of particles. ${ }^{6}$ Particles in a DEM simulation are considered to be rigid bodies interacting with each other by fictitious springs, dashpots and/or sliding blocks that simulate the contacts. At each time step considered in the transient analysis, particles move and new contacts are created and old contacts cease to exist. Figure 1 shows the development of contact between a pair of particles. The main assumptions considered in this method are: (1) particles are rigid elements and can move or rotate freely from one another; (2) contact occurs only between two particles, over an infinitesimal area; (3) particles may overlap slightly in the contacts, overlaps are considered to be small; (4) compression forces between particles can be calculated from interpenetration and tensile forces from particle separation (if particles are bonded); (5) the time step required to update particle's velocity and position should be small enough to ensure that, in a single time interval, perturbations will only propagate to the neighboring particles. The time step can be defined as:

$$
d t=\sqrt{\frac{m}{k}}
$$

where $m$ is the mass and $k$ is the stiffness of a system of particles. The contact forces developed in the interaction between particles can be calculated according to the contact law employed. The equations of normal and shear contact force vectors, for a linear interaction law, can be calculated respectively by: 


$$
F_{n}=k_{n} u_{n}
$$

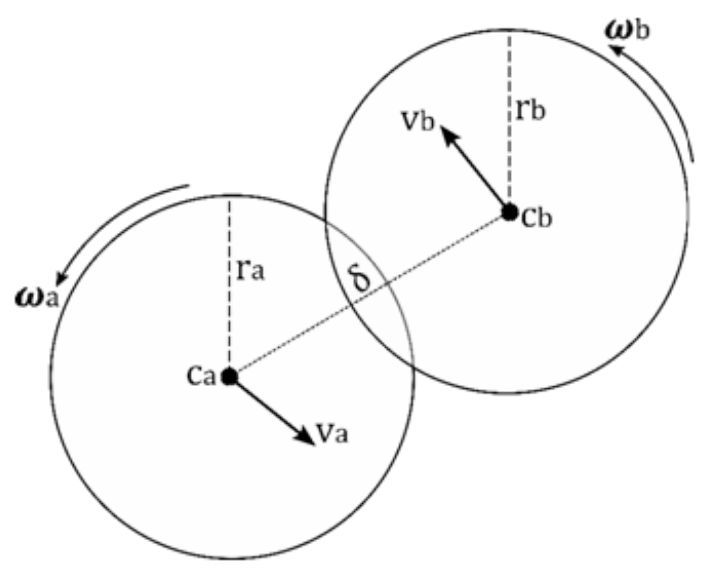

Figure I Development of contact between a pair of particles in a DEM simulation.

$$
\ddot{\mathrm{A}} F_{s}=-k_{s} \ddot{\mathrm{A}} u_{s}
$$

where $k_{n}$ and $k_{s}$ are the normal and tangential stiffness and $u_{n}$ and $u_{s}$ are the normal and tangential relative displacements. In addition, particle's dynamic behavior can also be determined from the translational and rotational acceleration vector defined, respectively, by:

Table I Parameters used in the reduced model

$$
\begin{aligned}
& \boldsymbol{F}=m(\boldsymbol{a}-\boldsymbol{g}) \\
& \boldsymbol{M}=I \ddot{\mathbf{E}}
\end{aligned}
$$

where $\boldsymbol{F}$ and $\boldsymbol{M}$ are the force and moment vectors, $I$ is the moment of inertia, $\boldsymbol{a}$ is the translational acceleration vector, $\ddot{\mathbf{E}}$ is the rotational acceleration vector and $g$ is the gravitational acceleration.

\section{Methodology}

The methodology adopted aimed the simulation with DEM using the software Yade to obtain parameters of the analyzed arrangements and to compare the results with the reduced model developed by Nakai. ${ }^{3}$

\section{Reduced model}

The research group of Nagoya Institute of Technology (NIT), led by professor Teruo Nakai, provided data on load testing on both shallow and deep foundations performed in idealized reduced models, shown in Figure 2, to be used for parametrization for the numerical tests. The reduced model consisted of an idealized soil composed with aluminum rods with radius of 1.6 and $3.0 \mathrm{~mm}$ and length of $50 \mathrm{~mm}$, distributed in the proportion of $3: 2$ by mass, resulting in a specific weight of $20.4 \mathrm{kN} / \mathrm{m}^{3}$. The aluminum rods were tested in a twodimensional confined compression test in order to find the parameters of the idealized soil. The other materials used to make the block and piles had their parameters supplied by manufacturers shown in Table 1.

\begin{tabular}{lllll}
\hline Element & Material & Young's Modulus (MPa) & Poisson's ratio & Porosity \\
\hline Idealized soil & Aluminum & 2 & 0,20 & 0,30 \\
Block & Aluminum & $7,03 \times 10^{15}$ & - & - \\
\hline
\end{tabular}

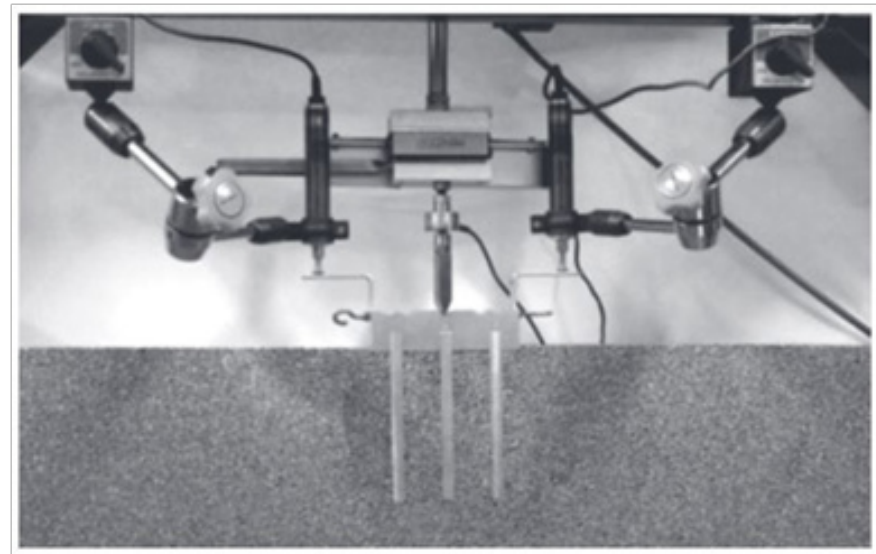

Figure 2 Load testing on deep foundation. ${ }^{3}$

\section{Numeric simulation}

The software used to conduct this research is known as Yade (Yet Another Dynamic Engine). Yade is an open source program included in the terminal installation package of Linux operating system. Its code was programmed in $\mathrm{C}++$, but its use is made via command terminal through scripts programmed in Python. Thus, Yade has become a very interesting tool for conducting research because it is free and easy to use.

\section{Sample generation}

Many researchers ${ }^{7-10}$ have proposed packing algorithms to generate soil samples. In this research, sample generation was done using the compaction method proposed by Jiang. ${ }^{11}$ Albuquerque ${ }^{12}$ proposed the following adaptations to the compaction method: (1) use of a single void ratio and (2) adoption of a slow decompression of each layer to avoid instabilities. These adaptations were used in this research in the sample generation script proposed by Albuquerque. ${ }^{8}$

The target porosity was determined by laboratory tests conducted by $\mathrm{Nakai}^{3}$, which allowed its calculation by means of resistance equations and void ratio correlations. The sample generation parameters can be seen in Table 2 .

Table 2 Sample generation parameters

\begin{tabular}{ll}
\hline Damping factor & 0 \\
\hline Sample compression velocity $(\mathrm{m} / \mathrm{s})$ & 5 \\
Dimensions & Length $=1 \mathrm{I}$ \\
Porosity & Height $=20$ \\
Number of particles & 0,22 \\
\hline
\end{tabular}

\section{Biaxial test}


After the sample was generated, a number of biaxial tests were done in order to find mesoscopic parameters that would reflect the macroscopic behavior of the sample, considering its initial conditions. To do so, properties at grains scale such as Young's modulus and friction angle of particles were varied to allow calibration of numerical and physical tests. Table 3 shows the parameters for sample generation.

Table 3 Sample generation parameters

\begin{tabular}{ll}
\hline Young's modulus $(\mathrm{MPa})$ & $80-100$ \\
\hline Contact friction angle $\left({ }^{\circ}\right)$ & $4-13$ \\
\hline
\end{tabular}

\section{Reduced model validation}

After the calibration of the numerical model, it was possible to obtain the grain-scale data required for the reduced model validation using DEM. It was performed using the same method of compaction of the sample generated for the biaxial test, however, the dimensions were adapted to correspond to the reduced model already presented. Table 4 presents the sample generation parameters. It should be noted that due to the change in laboratory porosity, it was necessary to conduct a brief calibration of the friction angle.

Table 4 Sample generation parameters

\begin{tabular}{ll}
\hline Parameter & Sample \\
\hline Damping factor & 0 \\
Sample compression velocity $(\mathrm{m} / \mathrm{s})$ & 5 \\
Dimensions & Length $=100$ \\
Porosity & Height $=50$ \\
Number of particles & 0,26 \\
\hline
\end{tabular}

\section{Results}

In the following subsections, numerical results obtained from DEM simulations are presented and compared to biaxial compression and reduced scale strip foundation tests performed in laboratory.

\section{Biaxial test}

The influence of mesoscopic parameters on results was evaluated. During the calibration it was observed that mesoscopic friction angle governed failure and Young's modulus controlled the linearelastic behavior, as expected. Figure $3 \&$ Figure 4 show the influence of Young's modulus and friction angle on the calibration of the biaxial test. Table 5 shows the parameters, after the calibration, that corresponded to the experimental curve.

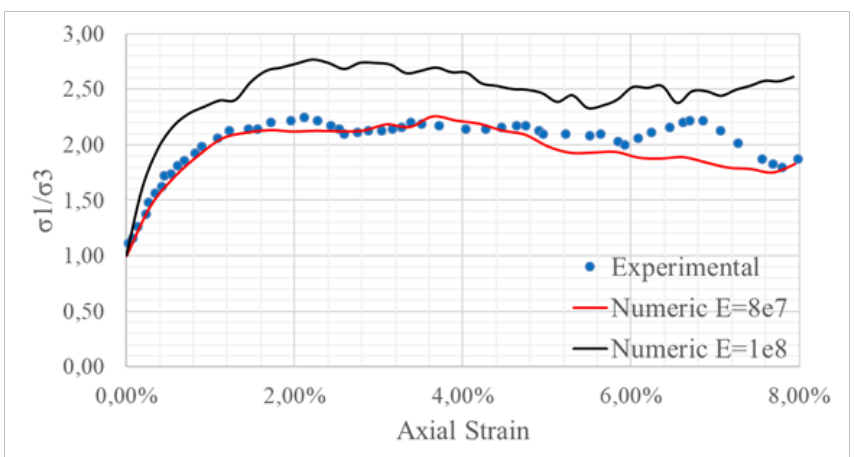

Figure 3 Influence of mesoscopic Young's modulus (E) on calibration of biaxial test.
The calculated macroscopic friction angle of the soil using MohrCoulomb failure criteria was $23^{\circ}$.

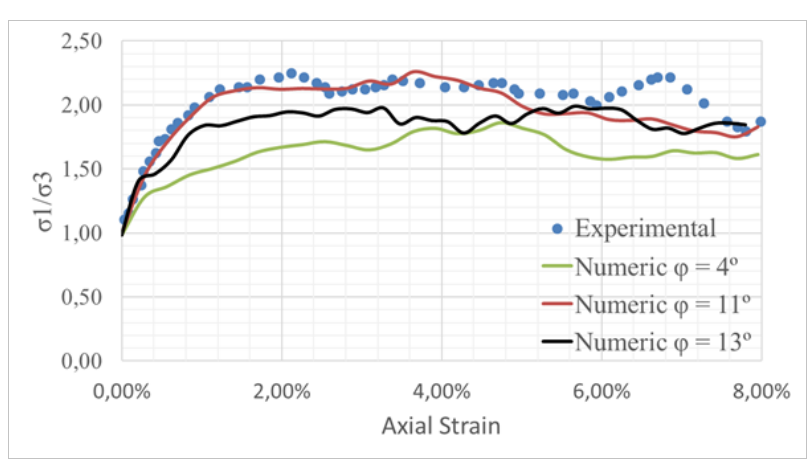

Figure 4 Influence of mesoscopic friction angle $(\varphi)$ on calibration of biaxial test.

Table 5 Sample generation parameters

\begin{tabular}{ll}
\hline Young's modulus $(\mathrm{MPa})$ & 80 \\
\hline Friction angle $\left({ }^{\circ}\right)$ & $\mathrm{II}$ \\
\hline
\end{tabular}

\section{Reduced model load testing}

From the calibration, numerical tests were conducted to reproduce a reduced model load testing of the shallow foundation (block) using Yade and calibrated parameters shown in Table 5. Figure 5 shows the results for the reduced model and numeric tests for the load testing. The axes were normalized by the specific weight of the soil $(\gamma)$ and the width of the block (B). Table 6 shows the parameters, after the calibration, that corresponded to the experimental curve. There was a change in the value of the contact friction angle between particles, due to the difference of porosity among the samples tested. Terzaghi analytical formulation and the ultimate load capacity obtained in the load testing of the reduced model were used to calculate the macroscopic friction angle of the sample. The obtained angle was very close to the calibrated angle, the value found was $25^{\circ}$. In addition, it was possible to observe the development of a failure mechanism very similar to that proposed by Terzaghi in his analytical formulation. Figure 6 shows the evolution of the block failure surface using DEM.

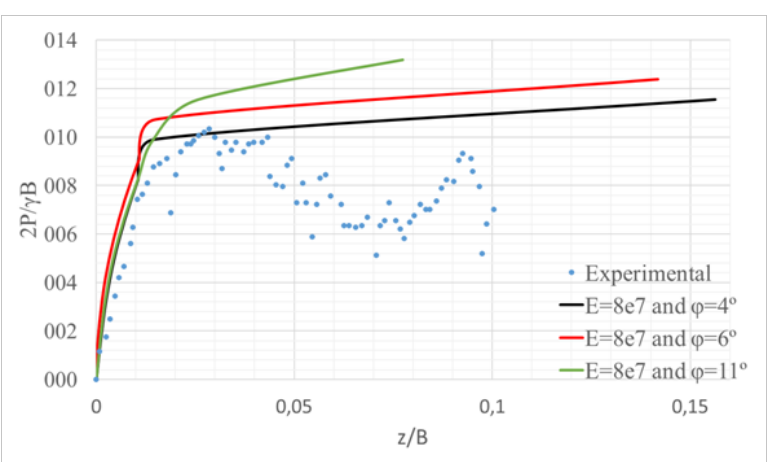

Figure 5 Load testing for the reduced model and numeric test with Yade.

Table 6 Sample generation parameters

\begin{tabular}{ll}
\hline Young's modulus $(\mathrm{MPa})$ & 80 \\
\hline Friction angle $\left(^{\circ}\right)$ & 4 \\
\hline
\end{tabular}

Citation: Rocha JSD, Farias MMD, Albuquerque BCPE, et al. Shallow foundation analysis using the discrete element method. Material Sci \& Eng. 2019;3(4):136-139. DOI: 10.15406/mseij.2019.03.00104 


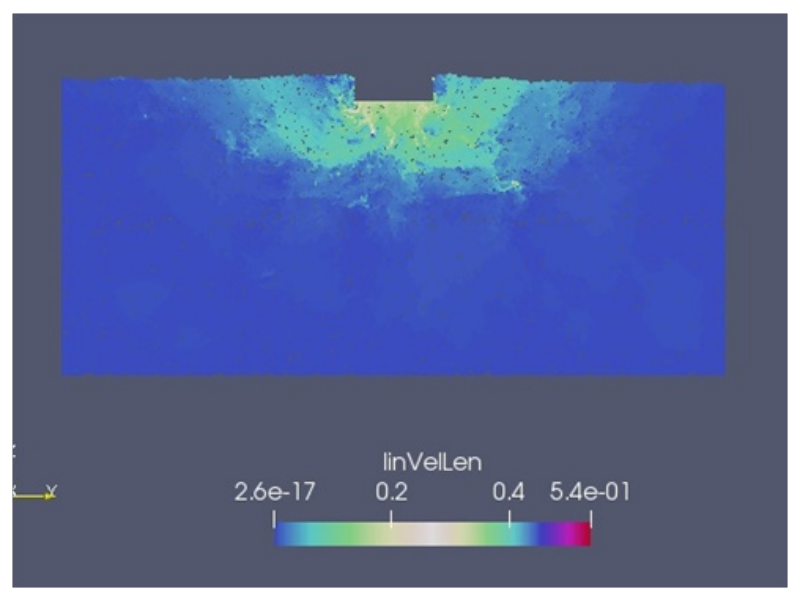

Figure 6 Velocity vector magnitude during block failure.

\section{Conclusion}

The discrete element method presents as an appropriate alternative for evaluating the behavior of reduced models of shallow foundations. Despite the need for calibration, the model provided an appropriate qualitative prediction of the failure mechanism and a satisfactory quantitative prediction of the failure load. In addition, the friction angle at which failure occurred in the biaxial test coincided with that of the reduced model test, despite the distinct conditions of those tests. The discrete element method is an efficient tool to evaluate the influence of mesoscale parameters on soil macroscopic behavior. As it was possible to be observed, the dominant mechanism in failure was governed by the friction angle between particles.

\section{Funding details}

None.

\section{Acknowledgements}

The authors of this research would like to thank professor Teruo
Nakai, Capes/CNPq and the University of Brasilia.

\section{Conflicts of interest}

Authors declare that there is no conflict of interest.

\section{References}

1. Wood DM. Geotechnical modelling. CRC Press; 2004.

2. Zuluaga RAG. Relationship between microstructural characteristics and macroscopic behavior of granular soils. University of Brasilia; 2016:171.

3. Nakai T. Constitutive modeling of geomaterials - principles and applications. CRC Press Taylor \& Francis Group; 2012.

4. De Bono J, McDowell GR. DEM of triaxial tests on crushable sand. Granular Matter. 2014;16(4):551-562.

5. Cundall PA, Strack ODL. A discrete numerical model for granular assemblies. Géotechnique. 1979;29(1):47-65.

6. O'Sullivan C. Particle-based discrete element modelling: geomechanics perspective. International Journal of Geomechanics. 2011;11(6):449-464.

7. Jiang M, Konrad J, Leroueil S, et al. An efficient technique for generating homogeneous specimens for DEM studies. Computers and Geotechnics. 2003;30:579-597.

8. Albuquerque BCP. Influence of the friction angle of the particle on the macroscopic resistance parameters of the medium in the deformation plane state. University of Brasilia; 2016:87.

9. Morfa CR, Cortés LA, Farias MM, et al. Systemic characterization and evaluation of particle packings as initial sets for discrete element simulations. Computational Particle Mechancis. 2017;5:319-334.

10. Morfa CR, Farias MM, Morales IPP, et al. Virtual modeling of polycrystalline structures of materials using particle packing algorithms and Laguerre cells. Computational Particle Mechanics. 2017;5: 213-226.

11. Jiang M, Konrad J, Leroueil S, et al. An efficient technique for generating homogeneous specimens for DEM studies. Computers and Geotechnics. 2003;30:579-597. 